\title{
A Connected-Components-Based Multi-scale Wavelet Approach for Wideband Spectrum Sensing
}

\author{
Chuandang Liu ${ }^{a}$, Luxi Lu ${ }^{b}$ and Guiliang Wang ${ }^{c}$ \\ National Key Lab of Science \& Technology on Blind Signal Processing, Chengdu 610041, China \\ aliuchuandang0603@126.com, byjluluxi@gmail.com, c13668146948@139.com
}

Keywords: Connected Component; Wideband Spectrum Sensing; Multi-scale Wavelet Transform; Spectrum Segmentation.

\begin{abstract}
This paper presents a new method for adaptive spectrum segmentation. In the field of wideband spectrum sensing, spectrum segmentation is regarded as the first procedure to detect sub-band signals. Wavelet Transform (WT) is sensitive to the rising and falling edges of signals in spectrum. Multi-scale WT can detect multi-width edges. The proposed method, based on the evaluation of the multi-scale WT, is designed for spectrum segmentation in multi-scale resolution FFT spectrum. Simulation results demonstrate that our method is validated on real satellite signals, and the results show good accuracy and flexibility of the sub-band signal detection.
\end{abstract}

\section{Introduction}

Wideband spectrum sensing is necessary for dynamic spectrum management in Cognitive Radios (CR). Spectrum segmentation is the significant first-step in wideband spectrum sensing. Conventional methods like energy detection, matched filtering, cycle stationary detection are all only suitable for narrow band signal detection and spectrum segmentation. [1] Sahai presents a method to regard wide band signal as several sequential narrow bands, but the time and hardware complexity is too high. [2] Therefore, Z. Tian etc. propose an algorithm based on Wavelet Transform (WT). [3] This algorithm obtains a much lower complexity but the signal model is so simplified that it cannot be used to handle real satellite signals. Almeida etc. improve Tian's algorithm by synthesizing multi-scale WT results, but the signal model is unknown. [4] G. Wang etc. propose another reforming algorithm based on Tian's but the algorithm cannot adapt to multi-resolution FFT spectrum. [5] Therefore, the algorithm application is very limited, though it obtains a really low segmentation missing rate about $5 \%$. Besides, D. Bao etc. propose a histogram based spectrum segmentation method, but the weakness is also obvious that all vital thresholds are not adaptive. [6]

This paper presents a new method for automatic spectrum segmentation. In preprocessing step, we redefine several vital thresholds to make them more adaptive. The most important innovation of our work is that we adopt a recursive connected component judgment in adjacent wavelet orders to handle multi-scale WT results so that our method can deal with multiple resolutions FFT spectrum circumstances.

The remainder of this paper is organized as follows. Problem for spectrum sensing is formulated in section 2. In section 3, we introduce the basic theory of wavelet transform. Section 4 emphasizes the spectrum segmentation processing flow. Section 5 illustrates the method performance and simulation results. Conclusion is finally presented in Section 6.

\section{Problem Formulation for Spectrum Sensing}

Suppose that there is a total of $\mathrm{BHz}$ band available in the wideband spectrum with frequency range $\left[f_{0}, f_{N}\right]$ for all devices in the wireless network. A CR at a specific place and time should be able to find out malicious users and sense the spectrum for detecting signals and spectrum holes. Suppose that some signal received by the CR occupies $N$ spectrum bands and these spectrum bands are located in frequency range $\left[f_{0}, f_{N}\right]$ consecutively. We use $f_{0}<f_{1}<\cdots<f_{N}$ to denote the 
frequency boundaries in $\left[f_{0}, f_{N}\right]$. Thus, the $n$-th band is defined by $B_{n}:\left\{f \in B_{n}: f_{n-1} \leq f<f_{n}\right\}$, $n=1,2, \ldots, N$. Fig. 1 shows a Power Spectral Density (PSD) structure of a wideband spectrum. However, the figure of real signal in spectrum cannot be rectangular because of utilization of imperfect filters in pulse shaping. Rising and falling edges of signals are continuously differentiable. Fig. 2 illustrates the PSD of a real signal.

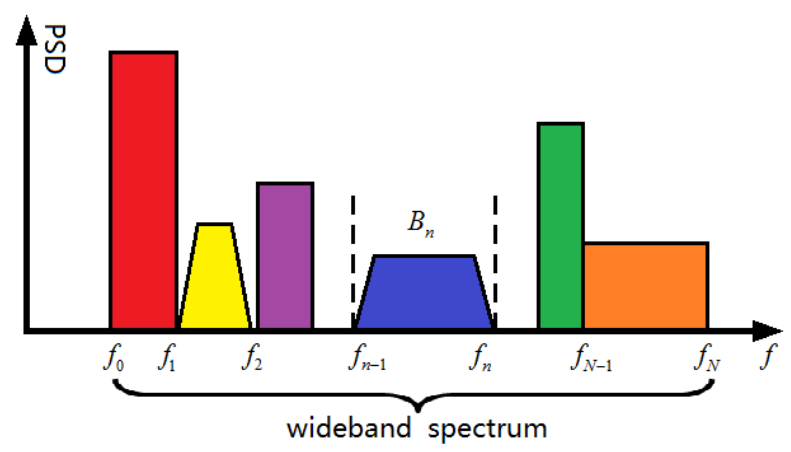

Fig. 1 Power Spectral Density (PSD) Structure of a Wideband Spectrum

Define indicator $I_{n}(f)$ as

$I_{n}(f)=\left\{\begin{array}{l}1, \forall f \in B_{n} \\ 0, \forall f \notin B_{n}\end{array}\right.$

Thus, the PSD of the signal in wideband spectrum can be written as

$S_{r}(f)=\sum_{n=1}^{N} \alpha_{n}^{2} I_{n}(f)+S_{w}(f), f \in\left[f_{0}, f_{N}\right]$

Where, $S_{r}(f)$ denotes the PSD of received signal, $S_{w}(f)$ denotes the PSD of white noise, and $\alpha_{n}^{2}$ indicates the signal power density within the n-th band $\left[f_{n-1}, f_{n}\right]$.

The wideband spectrum sensing problem is formulated as how to estimate parameters $N$ and $\left\{I_{n}(f)\right\}_{n=1}^{N}$ in $(2)$.

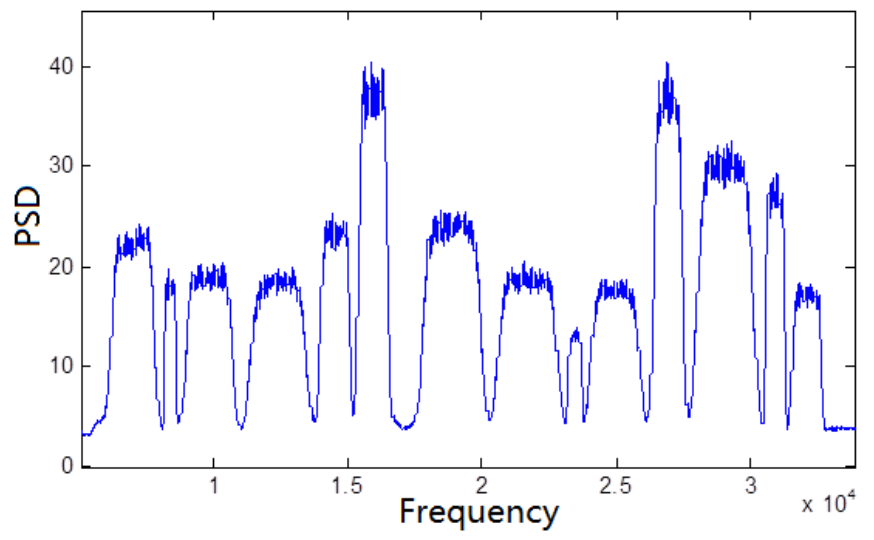

Fig. 2 PSD of a Real Signal in Wideband Spectrum

\section{Multi-Scale Wavelet Transform}

Wavelet Transform (WT) is proposed and consummated in 1986 when I. Daubechies etc. found a set of orthogonal wavelet basis. [7] There has been considerable research on wavelet analysis for time series and images. Singularity detection and processing with wavelets have been applied to filtering, denoising, compression, image processing and elsewhere.

Function $\theta(x)$ is called smoothing function if it fulfills the following conditions 


$$
\left\{\begin{array}{l}
\int_{-\infty}^{+\infty} \theta(x) d x=1 \\
\lim _{|x| \rightarrow \infty} \theta(x)=0
\end{array}\right.
$$

Let $\psi(x)$ be the first-order derivative of $\theta(x)$, i.e. $\psi(x)=d \theta(x) / d x$. According to the definition of smoothing function and wavelet function, $\psi(x)$ can be used as wavelet function.

The dilation of $\psi(x)$ by a scale factor $s$ is given by

$$
\psi_{s}(x)=\frac{1}{s} \psi\left(\frac{x}{s}\right)
$$

For any function $y=f(x)$, wavelet transform is the convolution of dilated wavelet function and function $y$ itself.

$$
W_{s} f(x)=\psi_{s}(x) * f(x)
$$

Substitute $\psi(x)=d \theta(x) / d x$ into (5), then we have

$$
W_{s} f(x)=\left(s \frac{d \theta_{s}(x)}{d x}\right) * f(x)=s \frac{d}{d x}\left(\theta_{s} * f\right)(x)
$$

Where $\theta_{s}(x)$ denotes the dilation of $\theta(x)$ by scale factor $s$.

Fig. 3 shows the convolution results. The two local maxima of $W_{s} f(x)$ locate at the positions where the only two steps of $f(x)$ locate. Therefore, we can find the local maxima of $W_{s} f(x)$ to make spectrum segmentation.

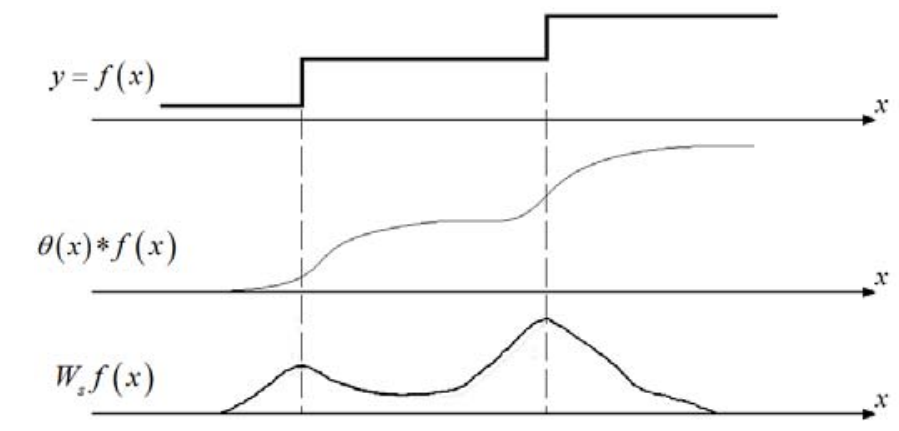

Fig. 3 Convolution with Smoothing Function and Wavelet Function

We can get multi-scale wavelet transform results by varying scale factor $s$.

\section{Spectrum Segmentation Method}

A basic phenomenon of multi-scale WT results is that if the signal occupies band $B$, then the local maxima of every convolution result will locate at band ends precisely on condition that the main lobe width of dilation wavelet function is less than $B$. An inference of convolution's linearity is that the responses of signals in convolution results are proportional to the power of signal. Therefore, we define two thresholds to filter multi-scale convolution results. One is bandwidth threshold which varies with scales of the wavelet function adaptively, and the other is an input power threshold that can filter small local maxima.

Let scale factor $s$ belong to a dyadic series $s \in\left\{2^{1}, 2^{2}, 2^{3}, \cdots\right\}$. We then compute convolution results of each factor and filter the results with thresholds. At last, we will obtain indicator matrix $I(s, f)$ of each scale factor $s$ within the whole wideband spectrum. Fig. 4 shows the test spectrum and the Indicator Matrix (IM) $I^{m \times n}$, in which $m$ denotes the number of scale factors 
$s \in\left\{2^{1}, 2^{2}, \cdots, 2^{m}\right\}$ and $n$ indicates the number of FFT points. Here in Fig. $4, n=2^{13}$ and $m=10$. Black blocks indicate $0 \mathrm{~s}$ in IM and the whites indicate $1 \mathrm{~s}$.

All of the right half section in row 10 (corresponding to scale factor $s=2^{10}$ ) is equal to 1 . So there is a problem that if the scale factor is relatively large (e.g. $s=2^{10}$ for $n=2^{13}$ points FFT), the method tends to regard a cluster of narrow signals as one wide signal. However, if the scale factor is relatively small, the method tends to detect small signals and white components in IM split into several branches.
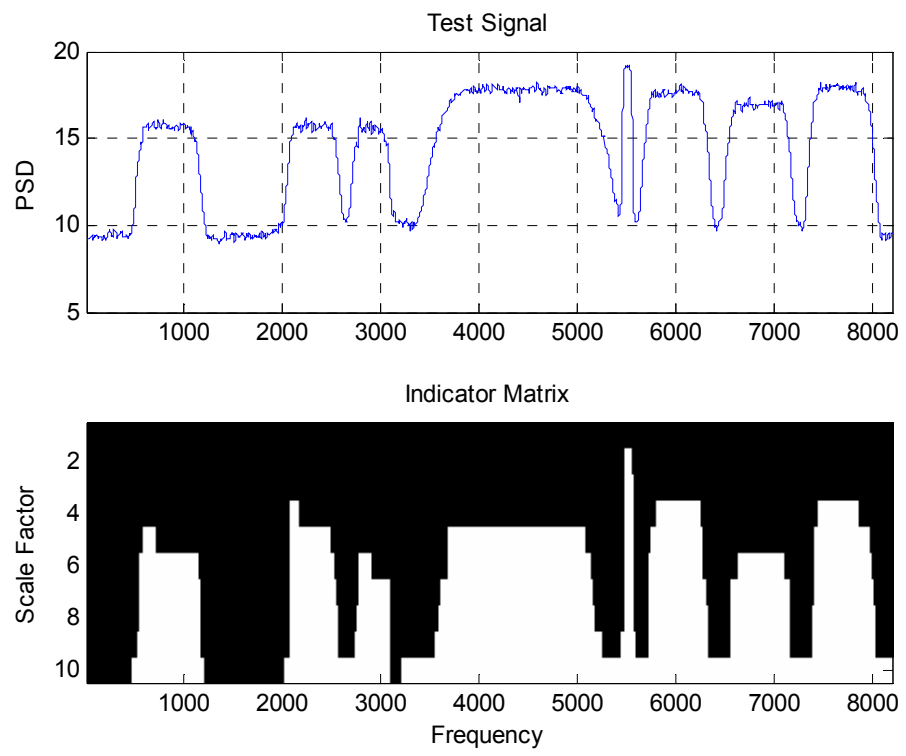

Fig. 4 Test Signal and Indicator Matrix

Total processing flow of spectrum segmentation method is illustrated in Fig. 5.

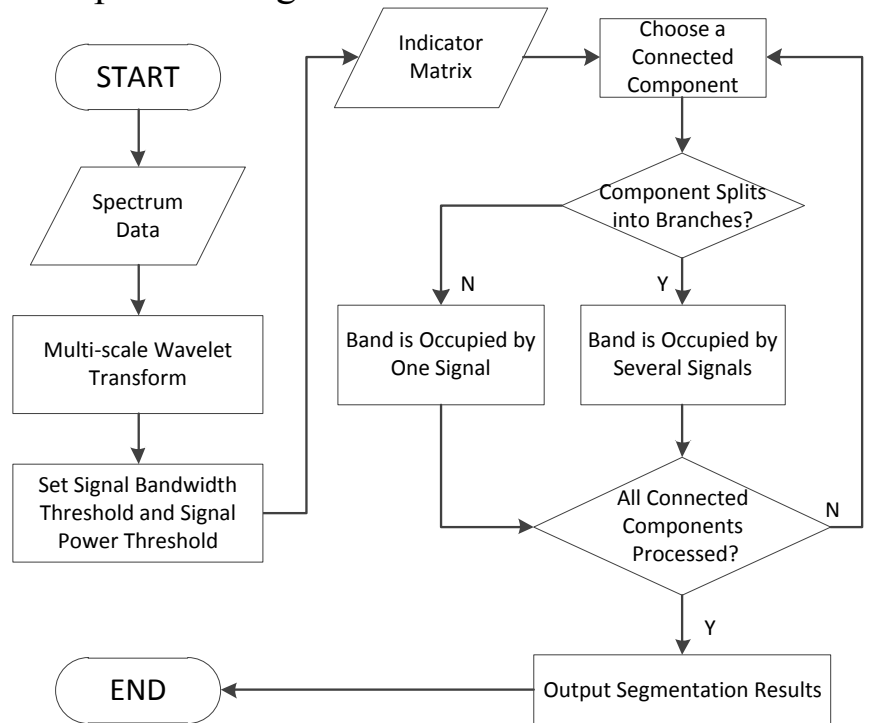

Fig. 5 Processing Flow of Spectrum Segmentation Method

Here, we adopt a recursive connected component judgment in adjacent wavelet scales to handle multi-scale WT results so that the method can deal with multi-resolution FFT spectrum scenarios. After getting indicator matrix, we use 4-connected neighborhood rule to find all connected components in matrix. Then for each component, we delete the greatest scale factor result and find out the number of connected components in the remainder to judge whether it splits into several branches. If the remainder splits, we recursively use the above method to handle each branch. Then for each component, we choose the bandwidth of median scale factor to segment spectrum. The red part in Fig. 6 shows the final segmentation result of test signal. 


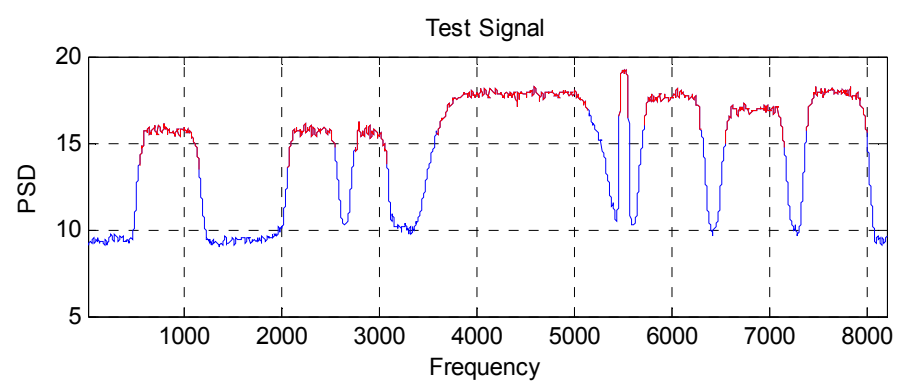

Fig. 6 Final Segmentation Result of Test Signal

\section{Performance Analysis and Simulation Results}

The definitions of Correct Rate $\left(R_{C}\right)$, Missing Rate $\left(R_{M}\right)$ and False Alarm Rate $\left(R_{F A}\right)$ are illustrated in Fig. 7 and formulated in (7).

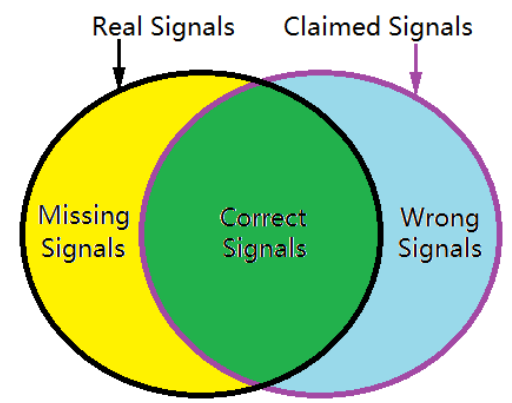

Fig. 7 Definitions of Correct Rate, Missing Rate and False Alarm Rate

$\left\{\begin{array}{l}R_{C}=\#\{\text { Correct Signals }\} / \#\{\text { Real Signals }\} \\ R_{M}=\#\{\text { Missing Signals }\} / \#\{\text { Real Signals }\}=1-R_{C} \\ R_{F A}=\#\{\text { Wrong Signals }\} / \#\{\text { Claimed Signals }\}\end{array}\right.$

To compare the performance of our method with witch are mentioned in section 1, we collect 83 satellite communication signal files which contain 1810 carriers (only counting carriers whose Carrier Noise Ratio (CNR) is greater than $3 \mathrm{~dB}$ ) in total. Each signal file is sampled with $93.3 \mathrm{MHz}$ and occupies $40 \mathrm{MHz}$ bandwidth.

G. Wang's algorithm can only handle $n \geq 2^{17}$ points FFT spectrum, so we calculate every signal file's FFT with $2^{17}$ points and make performance comparison. Table 1 shows the results of paper method and three others mentioned above.

D. Bao's algorithm contains some vital thresholds without computing methods, so we just try some thresholds and select the thresholds which leading to the best performance.

To emphasize the flexibility of FFT resolution, we then execute our method and draw performance curves along with increasing FFT orders.

Table 1 Spectrum Segmentation Results of Satellite Communication Signal ( $2^{17}$ FFT)

\begin{tabular}{cccc}
\hline Method & Correct Rate & Missing Rate & False Alarm Rate \\
\hline E. Almeida's & $91.88 \%$ & $8.12 \%$ & $5.78 \%$ \\
D. Bao's & $82.43 \%$ & $17.57 \%$ & $11.87 \%$ \\
G. Wang's & $96.63 \%$ & $3.37 \%$ & $4.06 \%$ \\
Ours & $95.80 \%$ & $4.20 \%$ & $2.97 \%$ \\
\hline
\end{tabular}

Fig. 8 shows the performance curve of correct rate and false alarm rate. We can see that along with increasing FFT order, correct rate curve increases rapidly at the beginning, but slowly when reach order 12. The reason is that signal file contains finite data amount and if FFT order is too high, the PSD calculation within the whole spectrum is not so accurate that curve increases slowly at high FFT orders. False alarm curve drops down monotonically along with the increasing FFT order. 


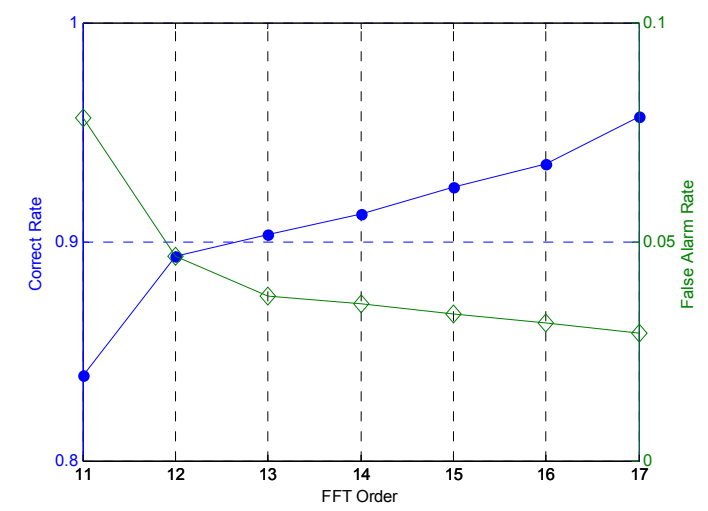

Fig. 8 Performance Curves of Proposed Method

\section{Conclusion}

In wideband spectrum sensing, spectrum segmentation is regarded as the first procedure to detect sub-band signals. In this paper, a new method for automatic spectrum segmentation is presented. The proposed method, based on the evaluation of the multi-scale Wavelet Transform (WT), is designed for spectrum segmentation in multi-scale resolution FFT spectrum. Simulation results demonstrate that our method is validated on real satellite signals, and the results show high accuracy and good flexibility of the sub-band signal detection and a processing complexity lower than the other methods.

Weakness of this method is also obvious that the bandwidth selection of median scale factor is not sufficiently reasonable. But the performance curve shows that this selection obtains adaptability while the spectrum segmentation accuracy doesn't get decreased.

Further work will be directed to a more precise signal segmentation method with a higher correct rate, a lower missing rate and a lower processing complexity.

\section{References}

[1]. Tevfik Yucek, Huseyin Arslan. A Survey of Spectrum Sensing Algorithms for Cognitive Radio Applications. IEEE Communications Surveys \& Tutorials. Vol. 11 (2009) No. 1, p.116-130.

[2]. A. Sahai, D. Cabric. Spectrum Sensing - Fundamental Limits and Practical Challenges. A tutorial presented at IEEE DySpan Conference. Baltimore, Nov. 2005.

[3]. Z. Tian, G.B. Giannakis. A wavelet approach to wideband spectrum sensing for cognitive radios. IEEE Int. Conf. on Cognitive Radio Oriented Wireless Networks and Communications. Mykonos, Jun. 2006, p.1-5.

[4]. E. Almeida, P. Carvalho, P. Cordeiro. Experimental Study of a Wavelet-based Spectrum Sensing Technique. 42nd Asilomar Conference on Signals, Systems and Computers. 2008, p.1552-1556.

[5]. G. Wang, Y. Lu, M. Han, G. Xu. A Multi-scale Wavelet Modulus Maxima Fusion Approach for Wideband Spectrum Sensing. Telecommunication Engineering. Vol. 51 (2011) No. 12, p. 63-67.

[6]. D. Bao, L. De Vito, S. Rapuano. A Histogram-Based Segmentation Method for Wideband Spectrum Sensing in Cognitive Radios. IEEE Trans. on Instrumentation and Measurement. Vol. 62 (2013) No. 7, p. 1900-1908.

[7]. I. Daubechies, A. Grossmann, Y. Meyer. Painless Nonorthogonal Expansions. Journal of Mathematical Physics. Vol. 27 (1986) No. 5, p. 1271-1283. 